\title{
Can a modified-simplified pulmonary embolism severity index ( $m$-sPESI) be used to predict the need for intensive care in hospitalized COVID-19 patients?
}

\author{
Ahmet Kagan As ${ }^{1}$. Burak Erdolu ${ }^{1}$. Burak Duman ${ }^{1} \cdot$ Elif Yazgan $^{1} \cdot$ Cuneyt Eris $^{1} \cdot$ Ufuk Aydin $^{1} \cdot$ Yusuf Ata ${ }^{1}$. \\ Ozlem Sengoren Dikis $^{2} \cdot$ Tamer Türk $^{1}$ (1)
}

Accepted: 16 February 2021 / Published online: 12 March 2021

(C) The Author(s), under exclusive licence to Springer Science+Business Media, LLC, part of Springer Nature 2021

\begin{abstract}
Severe acute respiratory syndrome-associated coronavirus-2 (SARS-CoV-2) causes coronavirus disease 2019 (COVID-19), which has been considered a pandemic by the World Health Organization (WHO). Clinical manifestations of COVID-19 disease may differ, most cases are mild, but a significant minority of patients may develop moderate to severe respiratory symptoms, with the most severe cases requiring intensive care and/or mechanical ventilatory support. In this study, we aimed to identify validity of our modified scoring system for foreseeing the approach to the COVID-19 patient and the disease, the treatment plan, the severity of morbidity and even the risk of mortality from the clinician's point of view. In this single center study, we examined the patients hospitalized with the diagnosis of COVID-19 between 01/04/2020 and 01/06/2020, of the 228 patients who were between 20 and 90 years of age, and whose polymerase chain reaction (PCR) tests of nasal and pharyngeal swab samples were positive. We evaulated 228 (92 male and 136 female) PCR (+) patients. Univariate analysis showed that advanced age $(\mathrm{p}<0.001)$, hemoglobin $(\mathrm{p}<0.001)$, troponin-I $(\mathrm{p}<0.001)$, C-reactive protein $(\mathrm{CRP})(\mathrm{p}<0.001)$, fibrinogen $(\mathrm{p}<0.001)$, HT $(\mathrm{p}=0.01)$, CAD $(\mathrm{p}=0.001)$, DM $(\mathrm{p}<0.001)$, history of malignancy $(\mathrm{p}=0.008)$, along with $\mathrm{m}$-sPESI scores $(\mathrm{p}<0.001)$ were significantly higher in patients that needed intensive care due to COVID-19 infection. In the multivariable logistic regression analysis, only the $\mathrm{m}$-sPESI score higher than $\geq 2$ was found to be highly significant in terms of indicating the need for ICU admission (AUC $0.948 ; 84.6 \%$ sensitivity and $94.6 \%$ specificity) $(\mathrm{p}<0.001$ ). With an increasing number of hospitalized patients, healthcare providers are confronting a deluge of lab results in the process of caring for COVID-19 patients. It is imperative to identify risk factors for mortality and morbidity development. The modified sPESI scoring system, which we put forward, is successful in predicting the course of the disease at the presentation of the patient with COVID-19 disease and predicting the need for intensive care with high specificity and sensitivity, can detect the need for intensive care with high specificity and sensitivity.
\end{abstract}

Keywords Covid-19 $\cdot$ Pulmonary thromboembolism $\cdot$ Thrombosis $\cdot$ PESI $\cdot$ SPESI $\cdot$ Intensive care unit

\section{Highlights}

Tamer Türk

tturkon@hotmail.com

1 Department of Cardiovasculer Surgery, University of Health Sciences, Bursa Yuksek Ihtisas Training and Research Hospital, Mimar Sinan Town, Emniyet Street, Yıldırım/ BURSA, Turkey

2 Department of Pulmonology, University of Health Sciences, Bursa Yuksek Ihtisas Training and Research Hospital, Bursa, Turkey
- COVID-19 is a disease with variable clinical manifestations. Although mild disease is the most common, mortality is quite high in the critical disease group.

- Simplified PESI (sPESI), which has been used for patients with pulmonary thromboembolism at the time of diagnosis for many years and provides scoring data in terms of possible mortality risk, is a good mortality predictor with its parameters and scoring system.

- We modified the scoring system (m-sPESI). We have detected that the m-sPESI scoring system will be useful 
in detecting need for intensive care with high specificity and sensitivity.

- This scoring system will be useful in detecting special situations such as the need for intensive care unit and predicting the course of the disease at the presentation of the patient with COVID-19 disease

\section{Introduction}

The new type of coronavirus infection (COVID-19), which first appeared in Wuhan province of China in December 2019, spread very rapidly, and affected the entire world. On March 11, 2020, the World Health Organization (WHO) declared that this disease, which created a health crisis, caused a pandemic. As of October 1, 2020, the balance sheet for COVID-19 is over 40 million patients, with an average mortality rate of $2.94 \%$ worldwide.

Clinical manifestations of COVID-19 disease differ. In fact, vastly different clinical presentations, such as asymptomatic carriage, mild upper respiratory tract symptoms, pneumonia with severe cough and dyspnea, sepsis, and multiorgan failure are encountered [1]. Although mild disease is the most common, mortality is quite high in the critical disease group. On average, the mortality rate is $2.3 \%$, however, this rate increases to around $50 \%$ in the critically ill patient group [2].

It has been determined that patients who show a severe course of the disease usually have various comorbidities, and numerous changes in some laboratory parameters can be associated with this disease [3]. In groups with a more severe course of the disease, some features such as male gender, advanced age, presence of underlying malignancy, cardiac disease, high D-dimer, high lactate dehydrogenase (LDH), and troponin elevation come to the fore. In patients with a prominent pneumonia, both damage to the lung vascular bed and necrosis of the lung parenchyma were observed [4]. All these findings are remarkably similar to that of pulmonary thromboembolism (PTE). Simplified PESI (sPESI), derived from the Pulmonary Embolism Severity Index (PESI), which has been used for patients with PTE at the time of diagnosis for many years and provides scoring data in terms of possible mortality risk, is a good mortality predictor with its parameters and scoring system [5]. Based on this similarity of COVID-19 infection with PTE, we examined the usability of sPESI as a predictor of clinical severity in COVID-19 patients at the time of initial presentation. We modified the critical age limit for COVID-19 patients as $>65$ years old, adapted it to the SPESI and created the modified-sPESI score $(m-s P E S I)$. The aim of this study is to predict the approach to the COVID-19 patient and the disease, the treatment plan, the severity of morbidity and even the risk of mortality from the clinician's point of view.

\section{Material and methods}

\section{Data sources}

Approval was obtained from the Turkish Republic Ministry of Health, General Directorate of Health Services, COVID-19 Scientific Research Evaluation Commission. Our study was approved by Bursa Yuksek Ihtisas Training and Research Hospital Clinical Research Ethics Committee dated 27/05/2020 and protocol numbered 2011-KAEK25 2020/05-16.

In this single center study, we examined the patients hospitalized with the diagnosis of COVID-19 in Bursa Yuksek Ihtisas Training and Research Hospital between $01 / 04 / 2020$ and 01/06/2020, who were between 20 and 90 years of age, and whose polymerase chain reaction (PCR) tests of nasal and pharyngeal swab samples were positive. Those who had major surgery/trauma in the last month, and a history of deep vein thrombosis and/ or pulmonary embolism were not included in the study. The study comprised a total of 228 patients who met the criteria. The laboratory values, radiological image data, demographic characteristics, measured vital parameters starting from the time of admission to the hospital, treatments and clinical outcomes of the patients were recorded.

\section{Potential variables}

Potential variables were recorded from the time the patients were first admitted to the hospital. Demographic data, age, gender, and smoking habits, the presence of hypertension, diabetes mellitus, chronic obstructive pulmonary disease (COPD), congestive heart failure (CHF), coronary artery disease (CAD), history of malignancy, renal failure, immunosuppression or use of immunosuppressants were recorded. Radiological images such as chest radiography and thoracic computed tomography (CT) were examined at the time of admission, and all normal or pathological data were noted. The clinical outcome, discharge information or death, duration of intensive care admission, and total hospital stay, vital signs, blood pressure, oxygen saturation $(\mathrm{SaO} 2)$, minute heart rate, laboratory data, complete blood count values (white blood cell count-WBC, hemoglobin-Hgb, neutrophil count, lymphocyte count, platelet count-PLT), biochemical examinations (ferritin, high sensitivity troponin-I, d-dimer, sedimentation, c-reactive protein (CRP)), blood urea nitrogen (BUN), creatinine, alanine amino transferase (ALT), aspartate aminotransferase (AST), lactate dehydrogenase (LDH) values were recorded, and the neutrophil/lymphocyte ratio (NLR) obtained by complete blood count was calculated and noted separately. 


\section{Analysis and interpretation of data}

The sPESI scoring used as a mortality predictor in patients with pulmonary thromboembolism (PTE) was modified (m-sPESI) and adapted to predict the clinical severity of COVID-19 disease. The score was obtained by processing the main variables, age, history of malignancy, chronic cardiopulmonary insufficiency (CPI), minute heart rate value (HR), systolic blood pressure value (SBP) and oxygen saturation (sPO2) values. The correlation between the patients' need for intensive care, hospital stay, mortality information and some laboratory results (CRP, NLR, D-dimer, troponin-I, etc.) were examined. Based on the meta-analyses conducted considering morbidity and mortality in COVID-19 and the Turkish Republic, Ministry of Health [6], we considered the risky age group as 65 years and above for sPESI, and not 80 years. With this change, the scoring system used in the study became a modified sPESI ( $m$-sPESI). In addition to this modification, we based the total score on the score of each parameter in m-sPESI. Unlike in sPESI, the presence of at least one of the parameters caused the score to be interpreted significantly. Table 1 shows SPESI and m-sPESI.

\section{Statistical methods}

SPSS 22.0 (IBM Statistical Package for the Social Sciences Statistic Inc. version 22.0, Chicago, IL, USA) was used for the analysis of statistical data. In our study, categorical variables were shown as frequency and percentage. Differences were investigated with Chi-square, Mann-Whitney U or Student $T$ test. Predictive value analysis wasperformed with Roc analysis for clinical data and scoring, and appropriate hemogram, biochemical parameters were investigated to show negative results. To show negative results, univariate analysis and multivariate logistic regression analysis were performed, as necessary.

\section{Results}

Of the 228 patients included in our study, 92 were male and 136 were female. The mean age of males and females were $54.28 \pm 16.15$ years and $53.21 \pm 17.06$ years, respectively. The demographic data of the patients included in the study are shown in Table 2.

In terms of clinical endpoints, there were significant differences in the data of patients who needed intensive care due to COVID-19 infection. As a result of univariate analysis of categorical variables, age $(69.65 \pm 14.27$ years

Table 1 sPESI and m-sPESI

\begin{tabular}{lllll}
\hline Parameters & sPESI & & m-sPESI & \\
\hline Age (years) & $80 \leq ; 0$ point & $80 \geq ; 1$ point & $65 \leq ; 0$ point & $65 \geq ; 1$ point \\
History of malignancy & No; 0 points & Yes; 1 point & No; 0 points & Yes; 1 point \\
History of cardiopulmonary diseases & No; 0 points & Yes; 1 point & No; 0 points & Yes; 1 point \\
Heart rate (min) & $<110 / \mathrm{min} ; 0$ points & $\geq 110 / \mathrm{min} ; 1$ point & $<110 / \mathrm{min} ; 0$ points & $\geq 110 / \mathrm{min} ; 1$ point \\
Systolic blood pressure $(\mathrm{mmHg})$ & $\geq 100 \mathrm{mmHg} ; 0$ points & $<100 \mathrm{mmHg} ; 1$ point & $\geq 100 \mathrm{mmHg} ; 0$ points & $<100 \mathrm{mmHg} ; 1$ point \\
Oxygen saturation $(\%)$ & $\geq 90 ; 0$ points & $<90 ; 1$ point & $\geq 90 ; 0$ points & $<90 ; 1$ point \\
\hline
\end{tabular}

sPESI = Zero (0) points; low mortality risk

sPESI > Zero (0) points; high mortality risk

Table 2 Demographic data

\begin{tabular}{ll}
\hline Female/Male, $\mathrm{n} / \mathrm{n}$ & $136 / 92$ \\
Mean age (years), female/male (mean $\pm \mathrm{sd})$ & $53.21 \pm 17.06 / 54.28 \pm 16.15$ \\
Diabetes mellitus, $\mathrm{n}(\%)$ & $45(\% 19.7)$ \\
Hypertension, $\mathrm{n}(\%)$ & $65(28.5)$ \\
Coronary artery disease, $\mathrm{n}(\%)$ & $33(\% 14.5)$ \\
Smoking, $\mathrm{n}(\%)$ & $52(\% 22.8)$ \\
Chronic obstructive pulmonary disease, $\mathrm{n}(\%)$ & $8(\% 3.8)$ \\
History of malignancy, $\mathrm{n}(\%)$ & $5(\% 2.2)$ \\
Chronic renal failure, $\mathrm{n}(\%)$ & $5(\% 2.2)$ \\
Presence of immune suppression, $\mathrm{n}(\%)$ & $2(\% 0.9)$ \\
Congestive heart failure, $\mathrm{n}(\%)$ & $12(\% 5.3)$ \\
\hline
\end{tabular}


vs $51.58 \pm 15.85$ years; $\mathrm{p}<0.001)$, hemoglobin $(11.20 \pm 3.40 \mathrm{~g} / \mathrm{dL}$ vs $12.89 \pm 1.75 \mathrm{~g} / \mathrm{dL} ; \mathrm{p}<0.001)$, troponin-I $(46.94 \pm 125.72 \mathrm{mg} / \mathrm{L}$ vs $7.97 \pm 18.75 \mathrm{mg} / \mathrm{L}$; $\mathrm{p}<0.001)$, C-reactive protein (CRP) $(92.30 \pm 61.87 \mathrm{mg} / \mathrm{L}$ vs $37.73 \pm 51.16 \mathrm{mg} / \mathrm{L} ; \mathrm{p}<0.001)$, fibrinogen $(654.192 \pm 199.619 \mathrm{mg} / \mathrm{dL}$ vs $463.35 \pm 199.35 \mathrm{mg} / \mathrm{dL}$; $\mathrm{p}<0.001)$, co-morbidities such as HT $(\mathrm{p}=0.01)$, CAD $(\mathrm{p}=0.001), \mathrm{DM}(\mathrm{p}<0.001)$, history of malignancy $(p=0.008)$, along with m-sPESI scores $(p<0.001)$ were significantly higher in patients that needed intensive care due to COVID-19 infection. The univariate analysis of variables of patients needing and not needing intensive care are presented in Table 3.

Since age, malignancy history, CPI history, HR, SBP and sPO2 are included in the m-sPESI scoring system, other parameters such as CRP, ferritin, fibrinogen, creatinine, neutrophil/lymphocyte, troponin, hemoglobin, presence of diabetes and m-sPESI score were included in multivariate analysis (Table 3), which revealed that the m-sPESI score was a highly significant independent variable for patients who needed intensive care hospitalization (OR 3.421 CI 95\%: 1.976-5.923, $\mathrm{p}<0.001)$. In the ROC analysis, m-sPESI scores $\geq 2$ and above were predictive of needing

Table 3 Univariate and multivariate analysis

\begin{tabular}{|c|c|c|c|c|c|}
\hline \multirow[t]{3}{*}{ Variables } & \multicolumn{3}{|c|}{ Univariate analysis } & \multicolumn{2}{|l|}{ Multivariate analysis } \\
\hline & \multirow{2}{*}{$\begin{array}{l}\operatorname{ICU}(-) \\
\mathrm{n}=202\end{array}$} & \multirow{2}{*}{$\begin{array}{l}\mathrm{ICU}(+) \\
\mathrm{n}=26\end{array}$} & \multirow[t]{2}{*}{$\mathrm{p}$} & \multirow{2}{*}{$\begin{array}{l}\text { OR (\%95 CI) } \\
\text { (lower-upper) }\end{array}$} & \multirow[t]{2}{*}{$\mathrm{p}$} \\
\hline & & & & & \\
\hline Mean age (years), female/male (mean \pm sd) & $51.58 \pm 15.85$ & $69.65 \pm 14.27$ & $<001$ & - & - \\
\hline Female/Male (n) & $121 / 81$ & $15 / 11$ & NS & - & - \\
\hline Smoking (n) & 47 & 5 & NS & - & - \\
\hline Hypertension (n) & 57 & 13 & 0.01 & - & - \\
\hline Coronary artery disease (n) & 23 & 10 & $<0.01$ & - & - \\
\hline Diabetes mellitus (n) & 33 & 12 & $<0.01$ & $0.533(0.130-2.187)$ & 0.382 \\
\hline COPD (n) & 5 & 3 & NS & - & - \\
\hline History of malignancy (n) & 3 & 2 & NS & - & - \\
\hline Chronic renal failure (n) & 1 & 4 & $<0.01$ & - & - \\
\hline Cardio pulmonary insufficiency (n) & 10 & 13 & $<0.01$ & - & - \\
\hline Presence of immune suppression (n) & 1 & 1 & $<0.01$ & - & - \\
\hline m-Spesi score (point) & $0.31 \pm 0.46$ & $0.95 \pm 0.20$ & $<0.01$ & $3.421(1.976-5.923)$ & $<0.001$ \\
\hline $\mathrm{WBC}\left(10^{3} / \mathrm{mm}^{3}\right)(\operatorname{mean} \pm \mathrm{sd})$ & $6.40 \pm 2.61$ & $8.07 \pm 4.65$ & NS & - & - \\
\hline Hemoglobin $(\mathrm{g} / \mathrm{dL})($ mean $\pm \mathrm{sd})$ & $12.89 \pm 1.75$ & $11.20 \pm 3.40$ & $<0.01$ & $0.775(0.536-1.122)$ & 0.177 \\
\hline Platelet $\left(10^{3} / \mathrm{mm}^{3}\right)($ mean $\pm \mathrm{sd})$ & $228.14 \pm 80.07$ & $233.80 \pm 128.97$ & NS & - & - \\
\hline Neutrophil $\left(10^{3} / \mathrm{ml}\right)($ mean $\pm \mathrm{sd})$ & $4.12 \pm 2.18$ & $6.39 \pm 4.34$ & $<0.01$ & - & - \\
\hline Lymphocyte $\left(10^{3} / \mathrm{ml}\right)($ mean $\pm \mathrm{sd})$ & $1.72 \pm 0.88$ & $1.06 \pm 0.65$ & $<0.01$ & - & - \\
\hline Neutrophil lymphocyte ratio (mean \pm sd) & $3.10 \pm 2.71$ & $7.03 \pm 4.61$ & $<0.01$ & $1.036(0.862-1.264)$ & 0.704 \\
\hline Ferritin $(\mathrm{mg} / \mathrm{L})($ mean $\pm \mathrm{sd})$ & $271.46 \pm 331.60$ & $720.75 \pm 725.29$ & $<0.01$ & $1.000(0.999-1.002)$ & 0.544 \\
\hline Troponin $(\mathrm{mg} / \mathrm{L})(\operatorname{mean} \pm \mathrm{sd})$ & $7.97 \pm 18.75$ & $46.94 \pm 125.72$ & $<0.01$ & $1.004(0.983-1.025)$ & 0.714 \\
\hline D-dimer $(\mathrm{ng} / \mathrm{mL})(\operatorname{mean} \pm \mathrm{sd})$ & $1.79 \pm 9.23$ & $2.60 \pm 3.47$ & NS & - & - \\
\hline Fibrinogen $(\mathrm{mg} / \mathrm{dL})($ mean $\pm \mathrm{sd})$ & $463.35 \pm 199.35$ & $654.19 \pm 199.61$ & $<0.01$ & 1.003 ( $1.000-1.007)$ & 0.087 \\
\hline Sedimentation $(\mathrm{mm} / \mathrm{h})(\mathrm{mean} \pm \mathrm{sd})$ & $35.19 \pm 25.08$ & $55.80 \pm 26.84$ & $<0.01$ & - & - \\
\hline C-reactive protein $(\mathrm{mg} / \mathrm{L})($ mean $\pm \mathrm{sd})$ & $37.73 \pm 51.16$ & $92.30 \pm 61.87$ & $<0.01$ & $0.997(0.984-1.010)$ & 0.648 \\
\hline Urea $(\mathrm{mg} / \mathrm{dL})(\mathrm{mean} \pm \mathrm{sd})$ & $13.00 \pm 5.51$ & $23.19 \pm 9.66$ & $<0.01$ & - & - \\
\hline Creatinine $(\mathrm{mg} / \mathrm{dL})($ mean $\pm \mathrm{sd})$ & $0.79 \pm 0.22$ & $1.08 \pm 0.46$ & $<0.01$ & $1.381(0.123-15.452)$ & 0.793 \\
\hline Aspartate aminotransferase (U/L) (mean $\pm \mathrm{sd})$ & $29.36 \pm 22.07$ & $36.73 \pm 18.84$ & NS & - & - \\
\hline Alanine aminotransferase (U/L) (mean $\pm \mathrm{sd})$ & $28.38 \pm 30.16$ & $26.84 \pm 17.20$ & NS & - & - \\
\hline Lactate dehydrogenase (U/L) (mean $\pm \mathrm{sd})$ & $253.01 \pm 96.62$ & $364.53 \pm 147.49$ & $<0.01$ & - & - \\
\hline Systolic blood pressure (mmHg) & $117.51 \pm 14.33$ & $86.84 \pm 11.00$ & $<0.01$ & - & - \\
\hline $\mathrm{sPO}_{2}(\%)$ & $97.00 \pm 2.84$ & $85.42 \pm 6.29$ & $<0.01$ & - & - \\
\hline Heart rate $(\min )$ & $86.05 \pm 15.38$ & $125.46 \pm 18.23$ & $<0.01$ & - & - \\
\hline
\end{tabular}

$N S$ nonspecific, $C O P D$ chronic obstructive pulmonary disease, $W B C$ white blood cells 
hospitalization in the intensive care unit (AUC $0.948 ; 84.6 \%$ sensitivity and $94.6 \%$ specificity) $(\mathrm{p}<0.001)$ (Table 3 ).

\section{Discussion}

The COVID-19 pandemic has caused an unexpected crisis in the world, especially for the health sector. As of 01 October 2020, the number of patients with COVID-19 infection in the world has exceeded 35 million and continues to increase. This number is also an indicator of the increased workload in health institutions. There is a great burden on the clinicians, especially the physicians who first meet with the patient, due to the high number of patients examined in health institutions. It has become particularly important to identify the patients as of the first application, to interpret the clinical findings and laboratory data correctly and to determine the effective diagnosis-follow-up-treatment strategy. With this study, we aimed to use a scoring system in which the clinician who welcomes the patient at the first admission can predict patient morbidity, in the light of the information and data that can be easily obtained from the patient.

The manifestation of COVID-19 infection has a wide spectrum, ranging from asymptomatic disease carrier to pneumonia with widespread lung involvement, multi-organ failure and sepsis. Although the majority of patients experience mild or moderate disease, in the study conducted by Guan et al., it was reported that the rate of severe disease and hospitalization was around 16\% [7]. The severity of the symptoms is also seen to vary from mild to severe. In addition to comorbidities such as HT, DM, CAD, immune deficiency or the presence of malignancy in the patient group with a particularly severe course of the disease, there are some changes in laboratory parameters such as d-dimer, high sensitive troponin-I, lactate dehydrogenase, ferritin, CRP that can be interpreted specifically for the disease [8]. Analyses in this severe disease group have shown that particularly patients in the intensive care unit are more prone to thrombosis and related complications [8, 9]. In addition, Tang et al. showed that abnormal changes in coagulation parameters in patients diagnosed with COVID-19 were associated with poor prognosis [8-12], and there was a significant reduction in mortality in COVID-19 patients who were treated with antithrombotic therapy [9-11]. In the first autopsy series examining pulmonary and cardiac pathologies in COVID-19-related deaths in New Orleans, USA, it was reported that thrombotic microangiopathies were encountered in the lung vascular bed, and especially fibrin deposits were observed in the distal and relatively thin parts of the pulmonary arteries. In addition, marked right ventricular dilatation was found along with cardiomegaly in all autopsies [13]. Similarly, in the first autopsy series of 80 cases reported by Edler et al., Germany, Hamburg, it was shown that the most common pulmonary complication accompanying COVID-19 pneumonia was PTE [14]. In their review, Giannis et al. stated this thrombotic process caused a tendency towards thrombosis and thrombotic complications in the lung vascular bed, not only in Sars-Cov-2, but also in Mers-Cov and Sars-Cov-1, which were also pandemic spreads, and that this thrombotic process was a lung-specific complication in coronavirus-related complications [15]. All these data showed us that the process is remarkably similar to PTE, especially in the moderate to severe-disease patient groups who require hospitalization.

Based on this similarity of COVID-19 infection with PTE, we established the m-sPESI scoring. All parameters included in sPESI pointed to the groups at risk for COVID19 infection. We only adapted the age parameter according to the 65-year age limit, which is considered risky for COVID-19, and thus modified the scoring. As of the beginning of the epidemic, in parallel with the increasing number of explosive cases all over the world, the number of patients who need an intensive care unit (ICU) bed has increased significantly. One of the regions that first felt this increasing ICU demand was Lombardy, Italy, where the elderly population was high [15]. However, in the pandemic scenarios created in Italy, patients who needed critical care could not be provided adequate service [16-18]. The situation was the same in similar scenarios adapted for the United States. If the isolation rules regarding the pandemic were strictly followed, the number of patients who needed intensive care would be decreased by $48 \%$, but even this figure was far above the intensive care capacity [19]. In a study in the USA in which 2490 hospitalized patients with COVID-19 infection were evaluated, ICU requirement and mortality data were analyzed. In that study, $32 \%$ of the patients needed intensive care, and in-hospital mortality rate was 17\% [20]. Similarly, in another study reported from China, the rate of need for intensive care was $32 \%$ and the mortality rate was $15 \%$ [21]. In a meta-analysis including 45 studies and 4203 patients, the need for ICU was $10.9 \%$ (95\% CI 4.5-19.3\%) and the mortality rate was $4.3 \%$ (95\% CI 1.0-9.1\%) [22]. In our study, the number of patients requiring intensive care was $26(11.4 \%)$, and 7 patients (3\%) died. As can be understood, there are serious differences between the studies in terms of ICU requirement and mortality. However, considering both the ICU need and the resulting mortality rates, the burden of COVID-19 infection on health systems is obvious.

The univariate analysis performed within the patient group in our study yielded that age, DM, HR, CRF, CPF, SBP, HR, m-sPESI and low sPO2 were effective in identifying patients in need of intensive care (Table 1). In many studies, parameters such as HT, DM, CPF, CRF, presence of immunosuppression, low sPO2 have been shown to be risk factors in terms of ICU need. CRP, ferritin, fibrinogen, creatinine, NLR, troponin-I, hemoglobin, and DM 


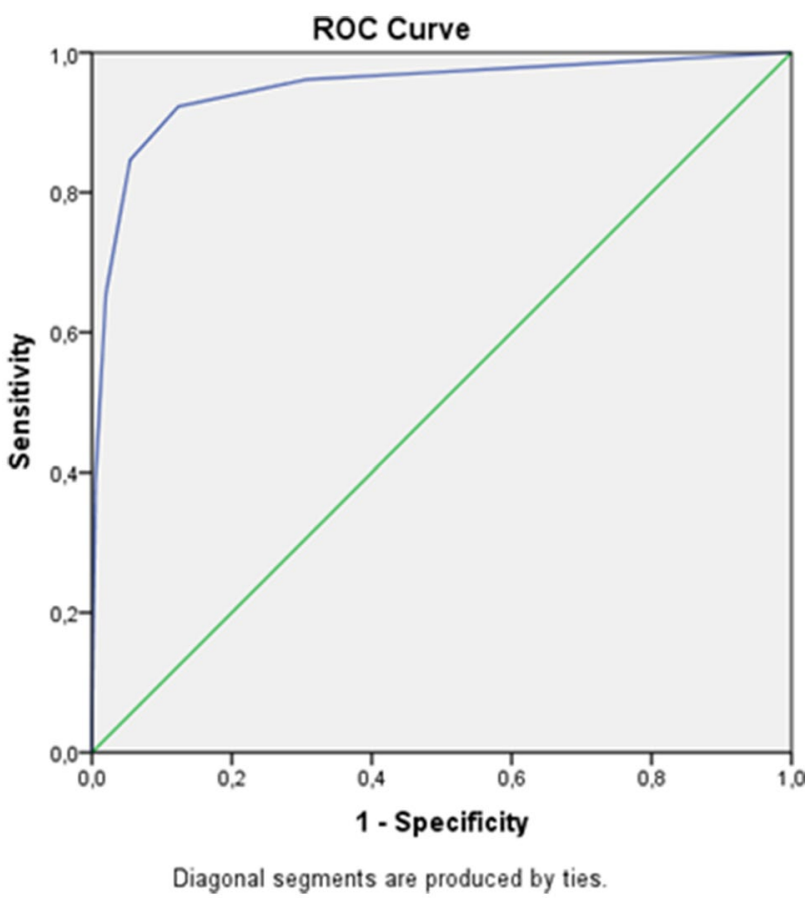

Fig. 1 Receiver operating characteristic (ROC) curve analysis for modified-simplified pulmonary embolism severity index

were evaluated by multivariate analysis, in which data that were within the parameters of the m-sPESI scoring system (age, cardiopulmonary disease, history of malignancy, heart rate, systolic blood pressure, and oxygen saturation) were not included (Table 3). In the multivariate logistic regression analysis, m-sPESI was an independent variable in showing the need for intensive care in COVID-19 patients, unlike the other analyzed parameters (OR 3.421 CI 95\% 1.976-5.923, p < 0.001). However, ROC analysis found that $\mathrm{m}$-sPESI was a strong predictor of intensive care need, especially for patients with a score $>2$ (AUC $0.948 ; 84.6 \%$ sensitivity and $94.6 \%$ specificity, $\mathrm{p}<0.001$ ) (Fig. 1). In our study, the m-sPESI scoring yielded statistically significant results in terms of predicting mortality. However, this statistically significant data was deliberately ignored, especially since more patients were needed to comment on mortality.

We have seen that there is no simplified scoring system in the approach to COVID-19 disease in the English literature review.

We updated the age-related scoring data of sPESI, which was previously proven to be effective in predicting mortality. We modified the age of 80 years, which was the limit value for sPESI, as 65 years for COVID-19, and put it into use as an effective protocol for predicting the need for intensive care with the name of the m-sPESI scoring system.

\section{Limitations}

The scope of this study consists of patients who were hospitalized with the diagnosis of COVID-19 and received treatment therefore, the number of patients is low. Although mortality data were statistically significant, the results were ignored due to the low number of deaths. The other significant limitation of the present study was retrospective study design. In addition, our study reports a single-center experience.

\section{Conclusion}

It is highly important for health systems struggling with the pandemic to determine the course of the disease early and take measures accordingly. Identifying morbidity development, the need for hospitalization, and especially the patients who need intensive care admission and close follow-up at presentation will ensure an equal distribution of the burden on health systems. The m-sPESI scoring system, which we put forward, will be useful in detecting special situations such as the need for intensive care with high specificity and sensitivity, and predicting the course of the disease at the presentation of the patient with COVID-19 disease.

Authors' contributions AKA: Substantial contributions to the conception or design of the work; or the acquisition, analysis, or interpretation of data for the work; drafting the work or revising it critically for important intellectual content; final approval of the version to be published. BE: Drafting the work or revising it critically for important intellectual content; final approval of the version to be published. BD: Drafting the work or revising it critically for important intellectual content; final approval of the version to be published. EY: Drafting the work or revising it critically for important intellectual content; final approval of the version to be published. CE: Drafting the work or revising it critically for important intellectual content; final approval of the version to be published. UA: Drafting the work or revising it critically for important intellectual content; final approval of the version to be published. YA: Drafting the work or revising it critically for important intellectual content; final approval of the version to be published. OSD: Interpretation of data for the work; drafting the work or revising it critically for important intellectual content; final approval of the version to be published. TT: Drafting the work or revising it critically for important intellectual content; final approval of the version to be published.

Funding None.

\section{Declarations}

Conflict of interest All authors declare that they have no conflict of interest.

Ethical approval The study was approved by Bursa Yuksek Ihtisas Training and Research Hospital Clinical Research Ethics Committee dated 27/05/2020 and protocol numbered 2011-KAEK-25 2020/05-16. 


\section{References}

1. Mizumoto K, Kagaya K, Zarebski A, Chowell G (2020) Estimating the asymptomatic proportion of coronavirus disease 2019 (COVID-19) cases on board the Diamond Princess cruise ship, Yokohama, Japan, 2020. Euro Surveill 25(10):2000180. https:// doi.org/10.2807/1560-7917

2. Wu Z, McGoogan JM (2020) Characteristics of and important lessons from the coronavirus disease 2019 (COVID-19) outbreak in China: summary of a report of 72314 cases from the Chinese Center for Disease Control and Prevention. JAMA 323(13):12391242. https://doi.org/10.1001/jama.2020.2648

3. Rodriguez-Morales AJ, Cardona-Ospina JA, Gutiérrez-Ocampo E et al (2020) Clinical, laboratory and imaging features of COVID19: a systematic review and meta-analysis. Travel Med Infect Dis. https://doi.org/10.1016/j.tmaid.2020.101623

4. Schaller T, Hirschbühl K, Burkhardt K, Braun G, Trepel M, Märkl B, Claus R (2020) Postmortem examination of patients with COVID-19. JAMA 323(24):2518-2520. https://doi.org/10.1001/ jama.2020.8907

5. Righini M, Roy PM, Meyer G, Verschuren F, Aujesky D, Le Gal G (2011) The Simplified Pulmonary Embolism Severity Index (PESI): validation of a clinical prognostic model for pulmonary embolism. J Thromb Haemost 9:2115-2117. https://doi.org/10.1 111/j.1538-7836.2011.04469.x

6. Zheng Z, Peng F, Xu B et al (2020) Risk factors of critical \& mortal COVID-19 cases: a systematic literature review and meta-analysis. J Infect 81(2):16-25. https://doi.org/10.1016/j. jinf.2020.04.021

7. Guan WJ, Ni ZY, Hu Y et al (2020) Clinical characteristics of coronavirus disease 2019 in China. N Engl J Med 382:1708-1720. https://doi.org/10.1056/NEJMoa2002032

8. Zhou F, Yu T, Du R et al (2020) Clinical course and risk factors for mortality of adult in patients with COVID-19 in Wuhan, China: a retrospective cohort study. The Lancet. https://doi. org/10.1016/S0140-6736(20)30566-3

9. Klok FA, Kruip MJHA, Van der Meer NJM et al (2020) Incidence of thrombotic complications in critically ill ICU patients with COVID-19. Thromb Res 191:145-147. https://doi.org/10.1016/j. thromres.2020.04.013

10. Tang N, Li D, Wang X, Sun Z (2020) Abnormal coagulation parameters are associated with poor prognosis in patients with novel coronavirus pneumonia. J Thromb Haemost 18(4):844-847. https://doi.org/10.1111/jth.14768

11. Tang N, Bai H, Chen X, Gong J, Li D, Sun Z (2020) Anticoagulant treatment is associated with decreased mortality in severe coronavirus disease 2019 patients with coagulopathy. J Thromb Haemost 18:1094-1099. https://doi.org/10.1111/jth.14817

12. Ari S, Can V, Demir ÖF, Ari H, Ağca FV, Melek M, Camci S, Sengoren Dikis O, Huysal K, Türk T (2020) Elevated eosinophil count is related with lower anti-factor Xa activity in COVID-19 patients. J Hematopathol 13:249-258. https://doi.org/10.1007/ s12308-020-00419-3

13. Fox SE, Akmatbekov A, Harbert JL et al (2020) Pulmonary and cardiac pathology in African American patients with COVID19: an autopsy series from New Orleans. Lancet Respir Med 8(7):681-686. https://doi.org/10.1016/S2213-2600(20)30243-5

14. Edler C, Schröder AS, Aepfelbacher M et al (2020) Dying with SARS-CoV-2 infection - an autopsy study of the first consecutive 80 cases in Hamburg, Germany. Int J Legal Med 134:1275-1284. https://doi.org/10.1007/s00414-020-02317-w

15. Giannis D, Ziogas IA, Gianni P (2020) Coagulation disorders in coronavirus infected patients: COVID-19, SARS-CoV-1, MERSCoV and lessons from the past. J Clin Virol 127:104362. https:// doi.org/10.1016/j.jcv.2020.104362

16. Grasselli G, Pesenti A, Cecconi M (2020) Critical care utilization for the COVID-19 outbreak in Lombardy, Italy: early experience and forecast during an emergency response. Jama 323(16):15451546. https://doi.org/10.1001/jama.2020.4031

17. Rodriguez Llanes JM, Castro Delgado R, Pedersen MG, Arcos Gonzalez P, Meneghini M (2020) Confronting COVID-19: surging critical care capacity in Italy. https://doi.org/10.2139/ ssrn. 3564386

18. Remuzzi A, Remuzzi G (2020) COVID-19 and Italy: what next? Lancet 395(10231):1225-1228

19. Moghadas SM, Shoukat A, Fitzpatrick MC et al (2020) Projecting hospital utilization during the COVID-19 outbreaks in the United States. PNAS 117(16):9122-9126. https://doi.org/10.1073/ pnas. 2004064117

20. Kim L, Garg S, O'Halloran A, et al (2020) Risk factors for intensive care unit admission and in-hospital mortality among hospitalized adults identified through the US coronavirus disease 2019 (COVID-19)-associated hospitalization surveillance network (COVID-NET) CID ciaa1012. https://doi.org/10.1093/cid/ciaa1 012

21. Huang C, Wang Y, Li X (2020) Clinical features of patients infected with 2019 novel coronavirus in Wuhan, China. Lancet 15(395):497-506. https://doi.org/10.1016/S0140-6736(20)30183 $-5$

22. Zhang JJY, Lee KS, Ang LW, Leo YS, Young BE (2020) Risk factors for severe disease and efficacy of treatment in patients infected with COVID-19: a systematic review, meta-analysis, and meta-regression analysis. Clin Infect Dis 71(16):2199-2206. https ://doi.org/10.1093/cid/ciaa576

Publisher's Note Springer Nature remains neutral with regard to jurisdictional claims in published maps and institutional affiliations. 